Conclusions Children with SN, present a patient history with a higher incidence of gestational-perinatal problems, genetic disorders and congenital heart diseases, thus presenting LD, $\mathrm{PD}$, a variety of behavioral and developmental disorders, with notable example the impaired hand dominance, mainly ambidexterity.

\section{P412 THE AGONY OF THE CHILD WITH COMPLEXITY: EXPLORING THE PSYCHOLOGICAL IMPACT ON PARENTS CARING FOR CHILDREN WITH COMPLEX HEALTHCARE NEEDS}

${ }^{1}$ Erica Crothers ${ }^{*},{ }^{2}$ Michelle Bradbury, ${ }^{1,2}$ Sarah Lewis, ${ }^{1,2}$ Clodagh O'Gorman, 'AnneMarie Murphy. ${ }^{1}$ Department of Paediatrics, University Hospital Limerick, Limerick, Ireland; ${ }^{2}$ Graduate Entry Medical School, University of Limerick, Limerick, Ireland

10.1136/archdischild-2019-epa.758

Aims Medical advances and improved neonatal care have led to increased survival of children with complex healthcare needs. The aim of this literature review was to explore what is known about the psychological impact on parents of caring for children with complex healthcare needs across North America and Europe, and more specifically within Ireland.

Methods A comprehensive search of the literature published within the last ten years was performed using PubMed and PyschINFO databases. Ten papers, consisting of qualitative studies and meta-analyses, which varied in sample size and methodology, were selected for detailed review.

Results Recent publications emphasise that caring for a child with complex care needs has a significant negative impact on the psychological wellbeing of parents. Several qualitative studies have shown that caregivers of children with life-limiting illness are up to twice as likely to report higher levels of parenting stress and depressive symptoms than parents of healthy children. The literature suggests that women experience greater parenting stress in caring for severely ill children than men, with one study noting an increase in maternal sick leave for psychological distress. However, women were more likely to report being the predominant caregiver, which could account for this discrepancy. Social isolation was a recurring factor contributing to the perceived burden of stress experienced by caregivers. Similar themes were evident across North America and Europe publications. We noted a paucity of research in the Irish context.

Conclusion Further research is needed to evaluate the psychological impact of caring for a child with complex healthcare needs and to guide management and prevention of parental stress and psychological illness, particularly in Ireland. Additional studies are needed to determine if increased social support reduces psychological stress and to investigate the effects of parental stress on disease progression in children with complex heathcare needs.

\section{P413 THE USE OF CEREBROLYSIN IN PERVASIVE DEVELOPMENTAL DISORDERS}

Aamir Al Mosawi*. Baghdad Medical City, Baghdad, Iraq

10.1136/archdischild-2019-epa.759

Introduction Pervasive developmental disorders (PDD) which has been increasingly called in the USA Autism spectrum disorder' include five chronic disorders marked by early impairment in socialization, communication, and repetitive behavior. There is no known curative therapy for PDD which include autism, Asperger syndrome, and atypical autism.

Objectives Marked improvement or disappearance of autistic features in these disorders have not been reported with any therapy before.The aim of this paper is report a retrospective observational study describing the use of a new therapeutic approach for the treatment of (PDD).

Methods During the year 2018, Six with PDD particularly autism and Asperger syndrome observed at the pediatric psychiatry clinic at the Teaching Hospital of Baghdad Medical City were treated with a new therapeutic approach which includes injectable cerebrolysin as the main therapeutic component. The patients ages ranged from 3 to 8 years.

The new approach aimed at improving the cardinal feature of PDD which is the impairment of social interaction which is mostly manifested by poor responsiveness to their name and infrequent engagement with others manifested by poor eye contact and infrequently looking to faces.

Most patient also required neuroleptics to control hyperactivity and other abnormal behaviors. Trifluoperazine andprochloperazine were used as necessary.

Some patients also received citicoline as an adjunctive therapy to improve speech development.

It is expected that improving social interaction will contribute to improving other features especially verbal communication and speech.

Courses of intramuscular cerebrolysin were given in individualized regimen depending on the age and severity of the illness and with aim of improving social interactions including response to name, looking at faces, and eye contact.

Results All the patients with autism and Asperger syndrome treated with this new approach showed improvement and marked lessening of the autistic features with some patients showed complete disappearance of the main autistic features. No patient developed any side effects.

Patients observed during the same year who didn't receive this treatment or were treated with other treatments such as omega-3 and risperdone didn't show any lessening effect in the autistic features. However, one patient treated with citicoline injection showed improvement in the autistic features. Conclusions Further studies are vital to study this new therapeutic approach.

\section{P424 A RARE CASE OF HYPERMETHIONINAEMIC ENCEPHALOPATHY IN AN INFANT WITH CLASSICAL HOMOCYSTINURIA}

1,2John Allen*, 'Bronwyn Power, 'Aida Abedin, 'Orla Purcell, 'Ina Knerr, ${ }^{1}$ Ahmad Monavari. ${ }^{1}$ Children's University Hospital Temple Street, Dublin, Ireland; ${ }^{2}$ Trinity College Dublin, Dublin, Ireland

10.1136/archdischild-2019-epa.760

Introduction Classical Homocystinuria is an autosomal recessive disorder caused by profound cystathionine $\beta$-synthase (CBS) deficiency. CBS deficiency is a disorder of metabolism of methionine leading to accumulation of homocysteine in both blood and urine. Clinical manifestations include lens dislocation, developmental delay, skeletal anomalies and thromboembolism. Treatment is lifelog and may include pyridoxine and specialised diet; medications such as betaine may also be considered as an adjunctive treatment. Betaine functions by 
donating a methyl group, thus converting homocysteine to methionine. Limited literature exists outlining the risk of metabolic encephalopathy associated with hypermethioninaemia presenting in children with classical Homocystinuria. Here we present one such case, made more remarkable by the absence of treatment with betaine.

Case description Our patient was diagnosed on newborn screening on day 4 of life with classical Homocystinuria and had been promptly commenced on a treatment regime. At four weeks of age he presented to his local hospital with poor feeding, vomiting, and lethargy. Following this acute presentation, he was started on IV antibiotics but a source of infection could not be established. Hyponatraemia, hypertension and proteinuria were noted. His renal ultrasound revealed increased echogenicity bilaterally with no evidence of renal vein thrombosis. His brain magnetic resonance imaging (MRI) showed areas of diffusion restriction bilaterally with normal MR spectroscopy and MR angiogram. Intensive care treatment was required, including intubation and ventilation, and antihypertensive treatment. No underlying thrombo-embolic cause could be established. His blood tests which were taken at the time of his acute presentation to his local hospital revealed a methionine of $1329 \mu \mathrm{mol} / \mathrm{L}$ with a normal cystine $(23 \mu \mathrm{mol} /$ $\mathrm{L})$ and a raised total homocysteine $(118 \mu \mathrm{mol} / \mathrm{L})$ and free homocysteine $(11 \mu \mathrm{mol} / \mathrm{L})$, it was therefore concluded that his presentation was metabolic in origin. These levels improved promptly with further natural protein restriction, increased calorie intake, including intravenous dextrose and electrolytes. The patient made a full recovery; his brain MRI findings normalised.

Discussion Hypermethioninaemic encephalopathy is a rare complication of classical Homocystinuria. It has previously been described in association with betaine treatment. We are unaware of any previous publications of this complication in patients not on this medication. In addition to monitoring for long-term complications, we suggest that methionine should be monitored regularly in patients with classical Homocystinuria, in particular during any episodes of acute deterioration.

\section{P425 SURGICAL MANAGEMENT OF MUCOPOLYSACCHARIDOSIS -RELATED SPINAL DEFORMITIES}

Polina Ochirova*, Sergey Ryabykh, Alexander Gubin. Russian Ilizarov Scientific Center, Kurgan, Russian Federation

\subsection{6/archdischild-2019-epa.761}

Study design Retrospective cohort study.

Class IV of evidence.

Introduction Spinal deformity in patients with mucopolysaccharidosis a problem requiring early treatment

Material and methods We analyzed 7cases of mucopolysaccharidosis (MPS), treated in Ilizarov Center in 2012 - 2018. There were 1 patient with MPS IH-type, 3 patients with IVAtype and 3 patients with MPS type VI. The age of the patients ranged from 6 to 26 years-old (average age - 10.3 years-old). All patients had spinal deformity with primary and/ or secondary stenosis of the spinal canal. Various surgical treatments were used: 1) two staged surgery was perfomed in one patient with cervical myelopathy and scoliosis: $\mathrm{C} 0-\mathrm{C} 7$ decompression with occipitospondylodesis (OSD) firstly and dual growing rod construction secondly; 2) final fusion in 2 patients with scoliosis; 3) decompression at the stenosis level (cervical spine) with OSD in 4 patients with cervical myelopathy.

Results Scoliosis value was from $20^{\circ}$ to $65^{\circ}$ Cobb, kyphosis from $15^{\circ}$ to $80^{\circ}$ Cobb. Four patients had vertebral stenosis and myelopathy (Frankel C). All patients had pulmonary and cardiac dysfunctions: vital capacity of the lungs was from $21 \%$ to $50 \%$ and abnormal ECG. Imbalance in the frontal and sagittal planes was present in $60 \%$ of patients. Through surgical treatment correction of the kyphosis was by $68 \%$ and scoliosis by $85 \%$. In $4(50 \%)$ patients with spinal stenosis neurologic status improved to Frankel E.

Conclusion Patients with MPS quite often have vertebral stenosis and myelopathy, in such patients with combined stenosis of the spinal canal, it is necessary to combine decompression and fusion.

\section{P426 PROFOUND METABOLIC ACIDOSIS AND HYPERTRIGLYCERIDAEMIA IN MITOCHONDRIAL 3- HYDROXY-3-METHYLGLUTARYL-COA (HMG-COA) SYNTHASE- 2 DEFICIENCY (HMGCS2D)}

${ }^{1}$ Tracey A Conlon*, ${ }^{2}$ Patricia E Fitzsimons, ${ }^{1}$ Abhidhamma Kaninde, ${ }^{2}$ Ingrid Borovickova, ${ }^{1}$ Ellen Crushell. 'National Centre for Inherited Metabolic Disorders, Children's University Hospital, Temple Street, Dublin, Ireland; 'Department of Paediatric Laboratory Medicine, Children's University Hospital, Temple Street, Dublin, Ireland

\subsection{6/archdischild-2019-epa.762}

Introduction Mitochondrial HMG-CoA synthase deficiency (HMGCS2D) is an autosomal recessive disorder of ketone body synthesis characterised by hypoketotic hypoglycaemia, vomiting and hepatomegaly typically in the first year of life.

Methods We report a previously well, 21-month old, unvaccinated male, of non-consanguineous Polish heritage, who presented with encephalopathy, hepatomegaly, severe anion-gap metabolic acidosis and mild hyperammonaemia following a brief intercurrent illness. While there was no documented hypoglycaemia, the sample was markedly lipaemic with Triglycerides 34 $\mathrm{mmol} / \mathrm{L}$. Unlike a similar, recently published case, he made a full neurological recovery.

Results Urine organic acid analysis revealed marked ketonuria, very marked dicarboxylic aciduria and notable increase in medium chain 3-oxodicarboxylic acids. Glutarate was markedly elevated with mild increase in 3-hydroxyglutarate (3HG). 4-hydroxy-6-methyl-2-pyrone and four other putative biomarkers for HMGCSD2 were detected. DBS acetylcarnitine was raised on presentation. Acidosis and hypertriglyceridaemia gradually resolved with intravenous dextrose and bicarbonate. 3HG was slow to normalize while pyrone remained detectable. Molecular genetic analysis of the HMGCS2 gene identified compound heterozygosity for c. $634 \mathrm{G}>\mathrm{A}$ and c. $1016+1 \mathrm{G}>\mathrm{A}$, not previously reported with this phenotype.

Conclusions HMGCS2D should be considered in a child with severe unexplained metabolic acidosis even without hypoglycaemia. The condition should be added to the differential diagnosis of raised urinary glutarate and $3 \mathrm{HG}$. 